\title{
Carnitine deficiency associated with long-term pivampicillin treatment: the effect of a replacement therapy regime
}

\author{
S.J. Rose, T.C. Stokes, S. Patel, M.B. Cooper'1, D.J. Betteridge' and J.E. Payne ${ }^{2}$ \\ Brook General Hospital, Shooters Hill Road, London SE18 4LW, 'Department of Medicine, University \\ College and Middlesex School of Medicine, London WC1E 6JJ, and ${ }^{2}$ Queen Mary's Hospital, Sidcup, \\ Kent, $U K$
}

\begin{abstract}
Summary: A 51 year old female developed a skeletal muscle myopathy after 3 months of pivampicillin therapy. Pivampicillin can cause carnitine deficiency due to the pivalic acid side group. Plasma carnitine content and the patients symptoms failed to improve significantly on discontinuing the drug. Oral carnitine replacement therapy was administered for 6 weeks but the patient's plasma carnitine levels responded only slowly to this treatment. It is suggested that pivampicillin inhibits uptake of carnitine from the gut and may either directly or indirectly depress endogenous carnitine synthesis. In such cases a more aggressive carnitine replacement regime is indicated and pivampicillin should be avoided in patients requiring long-term antibiotic administration.
\end{abstract}

\section{Introduction}

Carnitine is an essential co-factor for the use of fatty acids as a metabolic fuel. ${ }^{1}$ Abnormally high plasma carnitine content is associated with liver disease $^{2,3}$ whilst carnitine deficiency has been reported to cause skeletal myopathy, cardiomyopathy, vomiting, encephalopathy, hepatomegaly, hypoketotic glycaemia and a Reye's-like syndrome. ${ }^{4}$ Pivampicillin is known to cause carnitine deficiency in some cases through the action of pivalic acid which is conjugated to the penicillin moiety to increase absorption. ${ }^{5}$ In this study we report a patient with skeletal myopathy and severe carnitine deficiency following long-term pivampicillin treatment.

\section{Case report}

A 51 year old woman presented with a 6 month history of feeling unwell. Examination showed an enlarged spleen and splenic cyst. The spleen was removed and was found to contain granulomatous sarcoid with negative Ziehl-Nielson stain and negative culture for acid-alcohol fast bacilli. She was commenced on prednisolone with some improvement in her well-being. Ampicillin was given

Correspondence: T.C. Stokes, F.R.C.P.

Accepted: 22 April 1992 for 2 months as anti-pneumococcal prophylaxi but for the patient's convenience, pivampicillin at:-a dose of $250 \mathrm{mg}$ twice daily, was substituted. Three months after starting pivampicillin the patient complained of lethargy, muscle weakness and wasting, weight loss and poor appetite. Extensive investigations could not account for these symp- $\overrightarrow{\overrightarrow{0}}$ toms. After 5 months of pivampicillin therapy, 3 urinary and plasma carnitine levels were measur- $\overline{ }$ ed, ${ }^{6}$ and were found to be much lower than the normal range (see Table I). Pivampicillin treatment $\bar{O}$ was discontinued and plasma and urine carnitine 3 . content were measured at intervals of 10 days and $2 \frac{5}{3}$ months after the cessation of treatment and were found to have remained very low, although the $ᄋ$ patient had gained weight and her muscle bulk and power improved to some extent. The patient was $ᄋ$ treated with oral L-carnitine supplements, $1 \mathrm{~g} D$ daily. There was further clinical improvement but plasma and urine carnitine content did not app- N roach the normal range until after six weeks of treatment.

\section{Discussion}

Sepsis following splenectomy, caused by organisms $\stackrel{\oplus}{\stackrel{?}{?}}$ such as Streptococcus pneumoniae, Neisseria menin- $\bigcirc$ gitis, Escherichia coli and Haemophilus influenzae, $\stackrel{+}{+}$ is a well-recognized complication of the operation. 
Table I Plasma and urine carnitine content during pivampicillin treatment and carnitine replacement therapy

\begin{tabular}{|c|c|c|c|c|c|c|}
\hline & $\begin{array}{c}\text { During } \\
\text { pivampicillin } \\
\text { therapy }\end{array}$ & $\begin{array}{c}\text { Ten days } \\
\text { after stopping } \\
\text { pivampicillin }\end{array}$ & $\begin{array}{l}\text { Two months } \\
\text { after stopping } \\
\text { pivampicillin }\end{array}$ & $\begin{array}{l}\text { Post one } \\
\text { week course } \\
\text { carnitine }\end{array}$ & $\begin{array}{l}\text { Post six } \\
\text { week course } \\
\text { carnitine }\end{array}$ & $\begin{array}{c}\text { Normal } \\
\text { range }\end{array}$ \\
\hline \multicolumn{7}{|c|}{ Plasma levels $(\mathrm{nmol} / \mathrm{ml})$} \\
\hline Free carnitine & 5.4 & 4.6 & 2.0 & 1.8 & 13.1 & $22-50$ \\
\hline Short-chain esters & $<1.0$ & 0.7 & $<1.0$ & $<1.0$ & 8.2 & $3.5-10$ \\
\hline Long-chain esters & 2.6 & 3.0 & 2.9 & 0.7 & 2.6 & $0.6-2$ \\
\hline Total carnitine & 9.0 & 8.3 & 5.9 & 3.5 & 23.9 & $30-60$ \\
\hline \multicolumn{7}{|c|}{ Urine levels $(\mathrm{nmol} / \mathrm{mg}$ creatinine) } \\
\hline Free carnitine & 16.1 & 35.1 & 4.8 & 0.0 & $<1.0$ & $0-500$ \\
\hline Short-chain esters & 22.5 & 14.1 & 17.8 & 18.6 & 72.5 & $100-4000$ \\
\hline Total carnitine & 38.6 & 49.2 & 22.6 & 18.6 & 73.5 & $100-4500$ \\
\hline Patients weight (kg) & 58.0 & & & 62.0 & 62.5 & \\
\hline
\end{tabular}

Whilst there is doubt about the efficacy of anti-pneumococcal vaccines after splenectomy, particularly in immunocompromised patients, daily prophylactic penicillin adminstered for at least 2 years postoperatively has been found to be highly effective in preventing infection. ${ }^{7}$ In some cases, administration of pivampicillin has been shown to result in an immediate decrease in plasma carnitine content coupled with its excretion in the urine as pivaloylcarnitine,$^{8}$ due not to the antibiotic action of the drug but to the metabolism of the stabilizing pivalic acid group. This particular patient had been taking pivampicillin for 3 months before symptoms occurred. We have not found reports of other patients with severe carnitine deficiency presenting with symptoms of myopathy following long-term pivampicillin treatment.

The most surprising finding from this case study was the very long period for which carnitine deficiency persisted. Two months after the cessation of pivampicillin treatment, plasma total carnitine content was only $17 \%$ of the accepted normal value and had fallen significantly from the levels recorded during the administration of the antibiotic. Excretion of free carnitine in urine also fell during this period whilst the amount of shortchain esters excreted was maintained at a low but relatively constant level. Seven grams of carnitine taken orally over one week had no effect on raising the patient's very low plasma and urinary levels to normal values, and even after 6 weeks of this replacement regime plasma and urine carnitine content remained at the lower end of the normal range. In normal humans such an intake of carnitine leads to large increases in the plasma content. ${ }^{9}$ If, in this patient carnitine was being taken up in the gut at a normal rate, we would have expected to see increased urinary excretion as well as increased plasma content. This was not the case and leads us to suggest that at least one effect of pivalic acid is to inhibit carnitine uptake by this route. The body can synthesize its requirement for carnitine, however, the substrate for this process is trimethyllysine incorporated into protein. Since when pivampicillin was discontinued carnitine levels remained depressed, we also conclude that endogenous synthesis cannot make good the carnitine deficiency. This could be an indirect effect of pivalic acid rather than a direct action as the body is unlikely to tolerate the extent of protein breakdown required for endogenous carnitine biosynthesis for sustained periods.

Carnitine is well tolerated and doses of up to $15 \mathrm{~g} /$ day can be administered orally without serious side effects. ${ }^{10}$ We suggest that in similar cases more intensive oral carnitine replacement regime with, for example, $5 \mathrm{~g} /$ day or an intravenous administration regime is indicated. Pivalic acid derivatives of ampicillin are used to increase absorption, but should probably be avoided in patients requiring long-term administration.

\section{References}

1. Bremer, J. Carnitine structure and function. Physiol Rev 1983, 63: $1420-1478$.

2. Cooper, M.B., Forte, C.A., Round, M. et al. Plasma carnitine and alcohol induced liver damage. Med Sci Res 1987, 15: $671-673$.

3. Cooper, M.B., Forte, C.A., Hughes, R. \& Williams, R. Plasma carnitine content in acute (paracetamol-induced) liver damage. Med Sci Res 1991, 19: 287-288.

4. Editorial. Carnitine deficiency. Lancet 1990, 335: 631-633. 
5. Holme, E., Greter, J., Jacobsen, C.E. et al. Carnitine deficiency induced by pivampicillin and pivmecillam therapy. Lancet 1989, ii: 469-473.

6. Cederblad, G. \& Linstead, S. A method for the determination of carnitine in the picomole range. Clin Chim Acta 1971, 37: 235-243.

7. Zarrabi, M.H. \& Rosner, F. Rarity of failure of penicillin prophylaxis to prevent post-splenectomy sepsis. Arch Intern Med 1986, 146: 1207-1208.

8. Melegh, B., Kerner, J. \& Bieber, L.L. Pivampicillin promoted excretion of pivalocarnitine in humans. Biochem Pharmacol 1987, 36: 3405-3409.
9. Greig, C., Finch, K.M., Cooper, M.B. et al. The effect of oral supplementation with 1-carnitine on maximum and submaximum exercise capacity. Eur J Appl Physiol 1987, 56: 457-460.

10. Goa, K.L. \& Brogden, R.N. L-carnitine - a preliminary reivew of its pharmacokinetics and its therapeutic use in ischaemic cardiac disease and primary and secondary carnitine deficiencies in relationship to its role in fatty acid metabolism. Drugs 1987, 34: 1-24. 